\title{
New Strategy for Detection of Subclinical Coronary Atherosclerosis in Asymptomatic Patients With Type 2 Diabetes Based on Cardiac Multi-Detector Computed Tomography and Treadmill Test
}

\author{
Hae Chang Jeong, MD; Inna Kim, MD; Keun Ho Park, MD, PhD; Doo Sun Sim, MD, PhD; \\ Young Joon Hong, MD, PhD; Ju Han Kim, MD, PhD; Myung Ho Jeong, MD, PhD; \\ Jeong Gwan Cho, MD, PhD; Jong Chun Park, MD, PhD; Yun Hyeon Kim, MD, PhD; \\ Hyun Ju Seon, MD, PhD; Jae Dong Moon, MD, PhD; Youngkeun Ahn, MD, PhD
}

\begin{abstract}
Background: The current screening strategy for subclinical coronary atherosclerosis in asymptomatic diabetic patients is not sufficient in real clinical practice. A new strategy was investigated that uses cardiac multi-detector computed tomography (MDCT) and a treadmill test (TMT).
\end{abstract}

\begin{abstract}
Methods and Results: A total of 445 self-referred asymptomatic diabetic patients underwent cardiac MDCT. The treatment plan was determined according to the new strategy that uses MDCT and TMT. All patients underwent clinical follow-up and cardiac events were investigated. The incidence of subclinical atherosclerosis was $49.4 \%$. The group without plaque underwent clinical follow-up without treatment and did not experience any cardiac events in 675.1 person-years of follow-up. Among patients with subclinical atherosclerosis without significant stenosis $(n=136)$ who received medical treatment only, 11 patients experienced cardiac events over 326.4 person-years. The patients with significant stenosis $(n=84)$ underwent TMT. Patients with positive TMT $(n=14)$ underwent coronary angiograms and revascularization therapy was performed in all of them over 39.2 person-years. Patients with negative TMT $(n=70)$ underwent medical treatment, and 27 of them experienced cardiac events. The incidence of cardiac death was $0 \%$ during 3 years of follow-up.
\end{abstract}

Conclusions: The new strategy for detecting subclinical atherosclerosis on MDCT combined with TMT may be a useful method for minimizing the mortality rate from cardiovascular disease in asymptomatic diabetic patients. (Circ J 2014; 78: 671-678)

Key Words: Atherosclerosis; Diabetes mellitus; Multi-detector computed tomography

$\mathbf{T}$ ype 2 diabetes mellitus is associated with premature atherosclerosis ${ }^{1}$ and asymptomatic coronary artery disease (CAD), called silent ischemia. ${ }^{2}$ Previous studies have shown that $6-58 \%$ of asymptomatic patients with type 2 diabetes mellitus have CAD. ${ }^{3}$ Therefore, there is a clear clinical need to detect coronary atherosclerosis at an early stage in patients with type 2 diabetes who are at high risk of cardiac events.

Current American Diabetes Association guidelines recommend a treadmill test (TMT) for the early detection of CAD in asymptomatic patients with diabetes, ${ }^{4}$ but several studies have shown that the TMT can be associated with false-positive results, thus necessitating the use of follow-up imaging tests. ${ }^{5,6}$ Therefore, a screening strategy that uses TMT alone is not sufficient. In contrast, cardiac multi-detector computed tomography (MDCT) allows the accurate detection of coronary artery stenosis. In particular, the negative predictive value of MDCT has uniformly been found to be high, indicating that the technique may be most suitable as a non-invasive tool to rule out the presence of obstructive coronary lesions. ${ }^{7}$

Received August 18, 2013; revised manuscript received November 8, 2013; accepted November 12, 2013; released online January 7, 2014 Time for primary review: 30 days

Department of Cardiology (H.C.J., I.K., K.H.P., D.S.S., Y.J.H., J.H.K., M.H.J., J.G.C., J.C.P., Y.A.), Department of Radiology (Y.H.K., H.J.S.), Chonnam National University Hospital, Gwangju; and Health Promotion Center, Chonnam National University Hwasun Hospital, Hwasun (J.D.M.), Korea

The first two authors contributed equally to this work (H.C.J., I.K.).

Mailing address: Youngkeun Ahn, MD, PhD, FACC, FSCAI, Professor, Department of Cardiology, Cardiovascular Center, Chonnam National University Hospital, 671 Jebongro, Donggu, Gwangju 501-757, Korea. E-mail: cecilyk@ hanmail.net

ISSN-1346-9843 doi:10.1253/circj.CJ-13-1038

All rights are reserved to the Japanese Circulation Society. For permissions, please e-mail: cj@j-circ.or.jp 


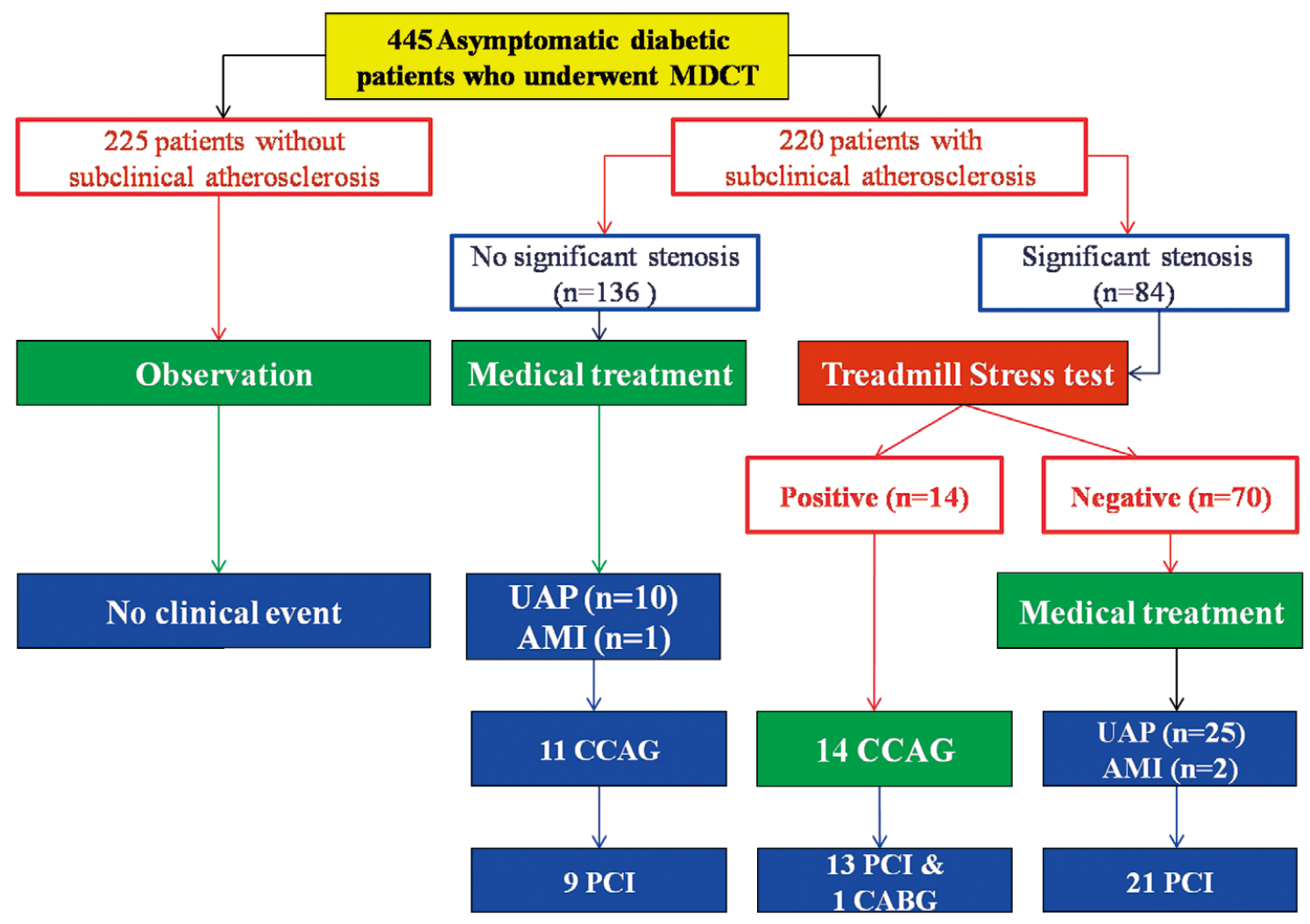

Figure 1. Study flow chart and clinical events in each study group. Clinical events are shown in bold and italic type. AMI, acute myocardial infarction; CABG, coronary artery bypass graft; CCAG, conventional coronary angiography; MDCT, multi-detector computed tomography; $\mathrm{PCl}$, percutaneous coronary intervention; UAP, unstable angina pectoris.

The present study evaluated the prevalence of subclinical atherosclerosis and coronary plaque characteristics in asymptomatic type 2 diabetic patients using MDCT, and investigated the predictors of subclinical atherosclerosis and adverse cardiac events. Also, we verified the effectiveness of a new screening strategy based on cardiac MDCT and TMT for minimizing the mortality rate from cardiovascular disease.

\section{Methods}

\section{Subjects}

We enrolled volunteers who visited the Health Promotion Center, Chonnam National University Hwasun Hospital, Korea and underwent MDCT from January 2007 to December 2011. The clinical indication for cardiac MDCT was a general medical check-up for assessment of CAD. The inclusion criteria were as follows: (1) age $\geq 18$ years; (2) diagnosis of type 2 diabetes mellitus; and (3) written informed consent. Patients were classified as having diabetes mellitus if they had an established diagnosis made by a physician and were receiving treatment with insulin or oral hypoglycemic agents. For the present study, we excluded patients with (1) history of heart disease, prior percutaneous coronary intervention (PCI), previous coronary artery bypass graft, or history of surgery on the heart or great vessels; (2) symptoms suspicious of cardiac disease or angina equivalent symptoms (dyspnea, chest pain, tightness, and pressure); (3) renal impairment (estimated glomerular filtration rate $\leq 60 \mathrm{ml} / \mathrm{min}$ ); or (4) cardiac MDCT images with poor quality or a low degree of confidence for stenosis and plaque characterization. Asymptomatic individuals free of known CAD were identified on the basis of patient history, which included the questionnaire and prior workup conducted by a physician.

\section{Study Design and Definitions}

For all subjects, we assessed clinical and diagnostic parameters, including medical history of hypertension and smoking, routine laboratory tests, and standard 12-lead electrocardiogram (ECG). Lipid profiles, fasting plasma glucose, glycated hemoglobin $\left(\mathrm{HbA}_{1 \mathrm{c}}\right)$, serum creatinine clearance level, and high-sensitivity C-reactive protein (hs-CRP) were measured. We also calculated the Framingham Risk Score (FRS) as per the Adult Treatment Panel III criteria. Each subject was then classified as having a low $(<10 \%)$, intermediate $(10-20 \%)$, or high $(>20 \%)$ risk of a CAD event over 10 years.

\section{Cardiac MDCT Acquisition and TMT}

Coronary artery calcium score (CACS) and coronary computed tomography angiography (cCTA) were done using a multidetector 64-channel row CT (Sensation Cardiac 64 scanner; Siemens, Forchheim, Germany). Before the scan, all subjects with a resting heart rate of $>70$ beats/min were given 50 $100 \mathrm{mg}$ oral metoprolol. Serial CT in the axial plane, together with an ECG-triggered examination, was performed from the level of the left ventricular apex after a bolus injection of $60 \mathrm{ml}$ non-ionic contrast media (Ultravist $370^{\circledR}$; Bayer Schering Pharma, Berlin, Germany) followed by a $60-\mathrm{ml}$ saline bolus 
injection, both of which were injected at a flow rate of $4 \mathrm{ml} / \mathrm{s}$. The scan parameters were as follows: $0.75-\mathrm{mm}$ section thickness with a gantry rotation time of $330 \mathrm{~ms}$ and a kernel value of B25f, $120-\mathrm{kV}$ tube voltage, and 800-mA tube current. The radiation dose was variable according to body mass index and heart rate: $0.8-1.3 \mathrm{mSv}$ in flash mode, $5-8 \mathrm{mSv}$ in sequence mode, and $10-13 \mathrm{mSv}$ in conventional mode.

All CT images were analyzed by a radiologist with 5 years' experience in coronary CT. The images were reconstructed by use of commercially available software (TeraRecon ${ }^{\circledR}$; iNtuition). CACS was measured with the use of the scoring system described previously by Agatston et al. ${ }^{8}$ In each coronary segment, coronary atherosclerosis was defined as the presence of any plaque. Plaque was classified into calcified, mixed, and non-calcified lesions. ${ }^{9}$ cCTA for each site was interpreted in accordance with the guidelines of the Society of Cardiovascular Computed Tomography, ${ }^{10}$ specifically, the 18-segment coronary artery tree model. The severity of stenosis was graded as normal (0-24\%), mild (25-49\%), moderate (50-74\%), and severe $(75 \%)$ narrowing. ${ }^{11}$ Significant stenosis was defined as $>75 \%$ of luminal narrowing.

Patients with significant stenosis on MDCT performed maximum exercise testing on a GE Marquette 15-Lead ECG Analysis/ GE Case T-2000 Series Treadmill System (GE Medical Systems, Milwaukee, WI, USA), according to the multistage Bruce protocol. The interpretation of the exercise testing results included the appearance of symptoms, exercise capability, and hemodynamic and ECG responses.

\section{Patient Management}

As described in Figure 1, the subjects were divided into 2 groups: those without any detectable plaque on MDCT and those with plaque. Patients without plaque received clinical follow-up only without any treatment for CAD. Patients with plaque were reclassified according to significant stenosis or insignificant stenosis of the coronary artery. The insignificant stenosis group received medical treatment only with antiplatelet drugs and vasodilators such as calcium channel blockers, nitrates, $\beta$-blockers, or nicorandil. If patients complained of CAD symptoms, we performed coronary angiogram or PCI if indicated by the angiographic findings. Patients in the significant stenosis group underwent TMT. Subjects with negative TMT received medical treatment and subjects with positive TMT underwent coronary angiogram and then PCI according to the angiographic findings.

We assessed cardiovascular events using a series of questions regarding intervening hospital admissions and reviewed the patients' medical records for hospitalizations that showed relevance for complaints of chest discomfort, dyspnea, vascular problems, cardiac problems, or any major surgery. A followup attempt was considered successful when surviving participants either returned to the clinic or completed a telephone interview and all relevant medical records were obtained. The study endpoint was cardiac events, which included (1) cardiac death; (2) non-fatal acute myocardial infarction (AMI) or unstable angina pectoris requiring hospitalization; and (3) revascularization regardless of symptoms because of positive TMT. We calculated the mean duration of follow-up as person-years (from the time of MDCT to the time of study end or incidence date of cardiac events in each person).

\section{Statistical Analysis}

SPSS for Windows, version 18.0 (Chicago, IL, USA) was used for all analyses. Continuous variables are presented as mean \pm $\mathrm{SD}$; categorical variables are presented as frequency and per-

\begin{tabular}{|c|c|}
\hline Characteristics & Data $(n=445)$ \\
\hline Age (years) & $58.2 \pm 8.3$ \\
\hline Male & $327(73.5)$ \\
\hline Duration of diabetes & $3.7 \pm 2.8$ \\
\hline \multicolumn{2}{|c|}{ Risk factors for coronary artery disease } \\
\hline Hypertension & $158(35.5)$ \\
\hline Current smoking & $90(20.4)$ \\
\hline Hyperlipidemia & $94(21.1)$ \\
\hline Family history of heart disease & $39(8.8)$ \\
\hline \multicolumn{2}{|l|}{ Laboratory findings } \\
\hline Total cholesterol (mg/dl) & $182.1 \pm 59.0$ \\
\hline Triglyceride (mg/dl) & $160.9 \pm 115.8$ \\
\hline $\mathrm{HDL}-\mathrm{C}(\mathrm{mg} / \mathrm{dl})$ & $46.3 \pm 11.7$ \\
\hline LDL-C (mg/dl) & $108.3 \pm 38.4$ \\
\hline Serum creatinine (mg/dl) & $1.0 \pm 1.2$ \\
\hline $\mathrm{HbA}_{1 \mathrm{c}}(\%)$ & $7.3 \pm 1.6$ \\
\hline Fasting glucose (mg/dl) & $143.7 \pm 48.4$ \\
\hline hs-CRP (mg/dl) & $1.0 \pm 7.2$ \\
\hline \multicolumn{2}{|l|}{ Echocardiography } \\
\hline LVEF (\%) & $67.3 \pm 5.6$ \\
\hline LVESD (mm) & $30.3 \pm 3.6$ \\
\hline LVEDD $(\mathrm{mm})$ & $48.8 \pm 3.7$ \\
\hline Fractional shortening (\%) & $35.8 \pm 25.9$ \\
\hline E/A ratio & $0.9 \pm 0.3$ \\
\hline $\mathrm{E} / \mathrm{E}^{\prime}$ ratio & $8.8 \pm 4.9$ \\
\hline \multicolumn{2}{|l|}{ Framingham risk score group } \\
\hline Low risk & $122(27.5)$ \\
\hline Intermediate risk & $232(52.1)$ \\
\hline High risk & $91(20.4)$ \\
\hline
\end{tabular}

Data given as mean \pm SD or $n(\%)$.

$\mathrm{HbA}_{1 \mathrm{c}}$, glycated hemoglobin; HDL-C, high-density lipoprotein cholesterol; hs-CRP, high-sensitivity C-reactive protein; LDL-C, low-density lipoprotein cholesterol; LVEDD, left ventricular enddiastolic dimension; LVEF, left ventricular ejection fraction; LVESD, left ventricular end-systolic dimension.

centage. For continuous variables, differences were investigated using Student's t-test. Discrete variables are presented as percentages and relative frequencies; comparisons were made using chi-squared test.

Multivariate logistic regression analysis was used to identify independent predictors of subclinical atherosclerosis, significant coronary stenosis on cCTA, and clinical events. The $95 \%$ confidence interval $(95 \% \mathrm{CI})$ for the relative risk was calculated using standard errors from the Kaplan-Meier curve. $\mathrm{P}<0.05$ was considered statistically significant.

\section{Results}

\section{Baseline Clinical Characteristics}

Overall, the subjects consisted of 445 patients with type 2 diabetes without angina or angina equivalent; $73.5 \%$ were male, and the mean age was 58.2 years. The mean duration of diabetes was 3.7 years. Among the subjects, 158 had hypertension, 94 had hyperlipidemia, and 90 were current smokers. Baseline echocardiogram was performed in all patients, which showed that the mean left ventricular ejection fraction was $67.3 \%$. No patients showed signs of myocardial ischemia. Of the 445 patients, $323(72.5 \%)$ were in the intermediate-high FRS group (Table 1). 
Table 2. Clinical Characteristics vs. Severity of Subclinical Atherosclerosis

Characteristics

Age (years)

Male

Hypertension

Current smoking

Laboratory findings:

Total cholesterol $(\mathrm{mg} / \mathrm{dl})$

Triglyceride (mg/dl)

HDL-C (mg/dl)

LDL-C (mg/dl)

Serum creatinine $(\mathrm{mg} / \mathrm{L})$

$\mathrm{HbA}_{1 \mathrm{c}}(\%)$

Fasting glucose (mg/dl)

hs-CRP (mg/L)

Framingham risk score

Medical treatment (\%)

\begin{tabular}{lc} 
Aspirin & $120(53.3)$ \\
$\beta$-blockers & $38(16.9)$ \\
Calcium channel blockers & $85(37.8)$ \\
Nitrates & $7(3.1)$ \\
Statins & $94(41.8)$ \\
\hline
\end{tabular}

Data given as mean \pm SD or $n(\%)$.
Abbreviations as in Table 1.

Data given as mean \pm SD or
Abbreviations as in Table 1 .

\section{No subclinical atherosclerosis $(n=225)$}

$56.8 \pm 7.8$

$153(68.0)$

69 (30.7)

30 (13.3)

$179.9 \pm 38.9$

$161.1 \pm 125.2$

$45.7 \pm 11.9$

$108.8 \pm 35.6$

$0.92 \pm 0.24$

$7.4 \pm 1.1$

$140.9 \pm 46.5$

$1.1 \pm 6.2$

$14.9 \pm 7.8$
Subclinical atherosclerosis without significant stenosis $(n=136)$

$59.4 \pm 8.8$
$104(76.5)$
$49(36.0)$
$36(26.5)$

$173.6 \pm 38.1$

$149.7 \pm 108.5$

$46.5 \pm 11.6$

$103.2 \pm 31.6$

$1.04 \pm 0.92$

$7.7 \pm 1.0$

$144.5 \pm 46.8$

$2.5 \pm 5.7$

$17.8 \pm 9.3$

$128(94.1)$

$65(47.8)$

$69(50.7)$

$29(21.3)$

$124(91.2)$
Subclinical

atherosclerosis

with significant

stenosis $(n=84)$

$\begin{array}{lr}60.6 \pm 8.6 & <0.001 \\ 70(83.3) & 0.016 \\ 40(47.6) & 0.021 \\ 24(28.6) & 0.001\end{array}$

$176.5 \pm 42.7$

0.398

$173.0 \pm 115.5$

0.392

0.839

0.398

0.109

0.008

0.442

0.008

$<0.001$

$21.0 \pm 11.4$

$82(97.6)$

$51(60.7)$

$68(80.9)$

$66(78.5)$

79 (94.0)
$<0.001$

$<0.001$

$<0.001$

$<0.001$

$<0.001$

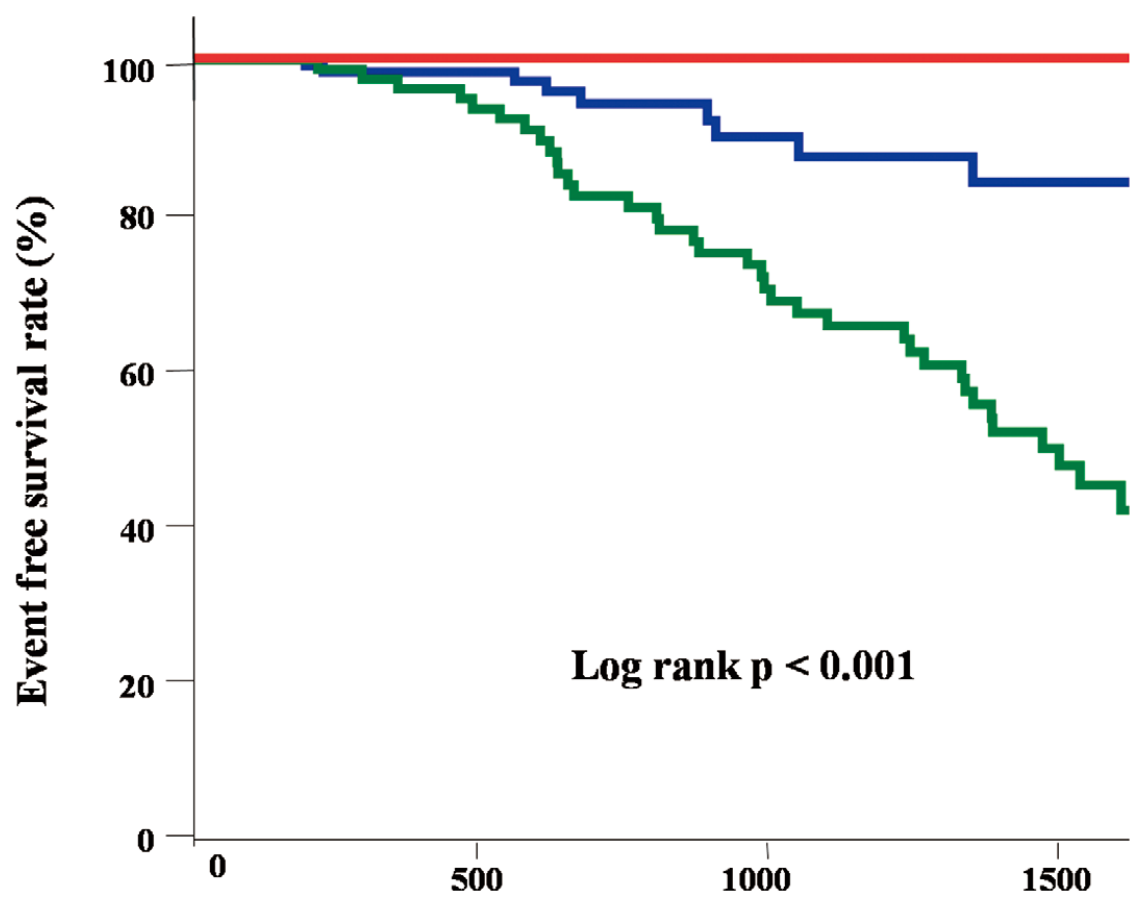

No subclinical

atherosclerosis group

Subclinical

atherosclerosis without significant stenosis group

Subclinical

atherosclerosis with significant stenosis group

\section{Follow up duration (Days)}

Figure 2. Kaplan-Meier curves for event-free survival rate. 


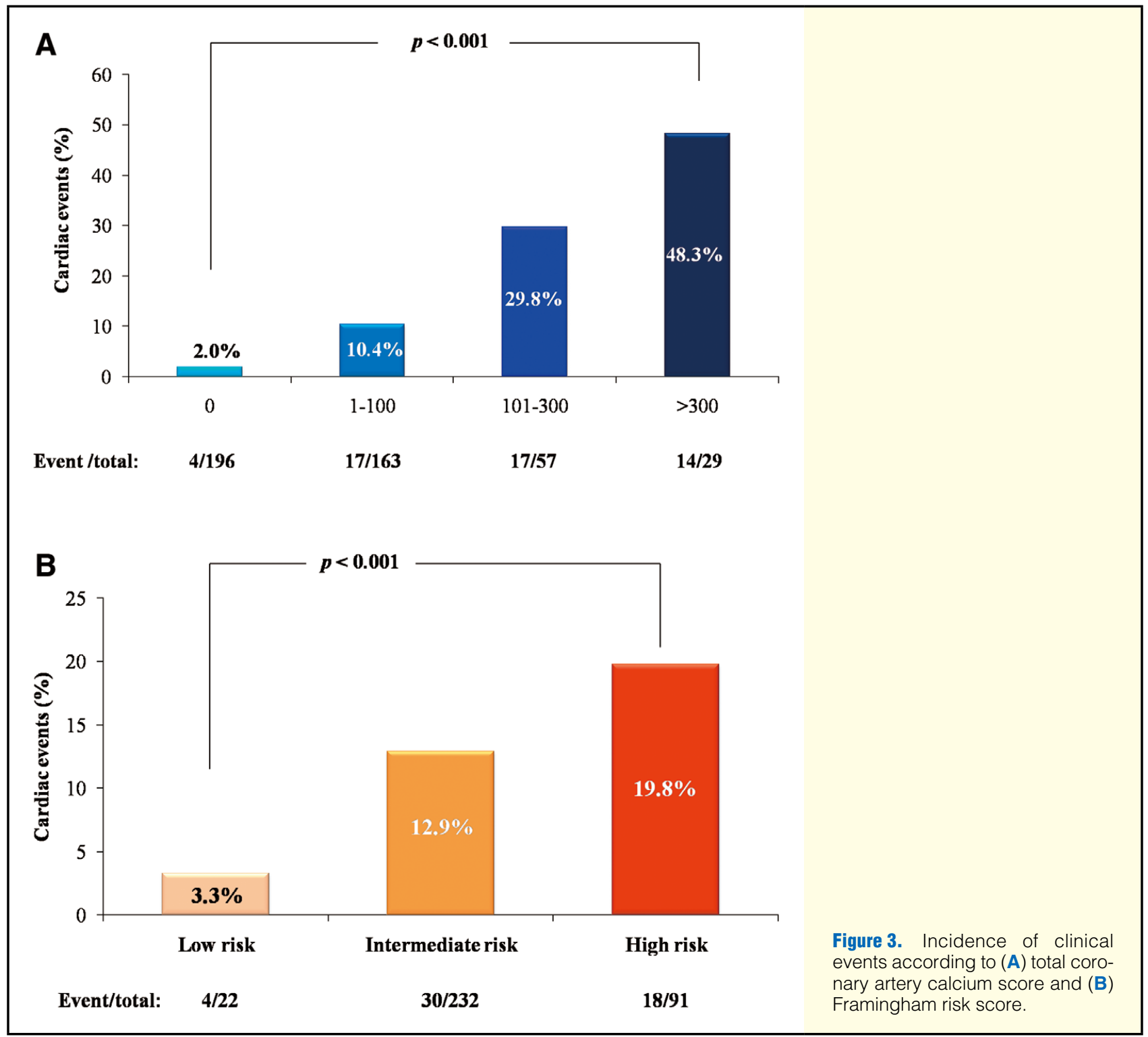

\section{MDCT Findings}

The mean total coronary artery calcium score (CACS) was 85.1 points. One hundred and ninety-six subjects $(44.0 \%)$ had total CACS 0, $163(36.7 \%)$ had CACS 1-100; $57(12.8 \%)$ had CACS $101-300$, and $29(6.5 \%)$ had CACS $>300$. Coronary calcium was most frequently detected in the left anterior descending artery $(47.6 \%)$. Plaque in the coronary arteries (subclinical atherosclerosis) was detected in 220 subjects (49.4\%). Mixed and calcified plaque were frequently observed. Significant luminal narrowing was found in 84 subjects (18.9\%). The most frequently involved vessel was the left anterior descending artery (16.2\%).

The patient clinical characteristics according to the presence of subclinical atherosclerosis and significant stenosis are given in Table 2. Patients in the subclinical atherosclerosis group were older, were more likely to be male, and were more likely to be current smokers. Also, $\mathrm{HbA}_{1 \mathrm{C}}$ and hs-CRP were significantly higher in the subclinical atherosclerosis group; patients in the group with significant stenosis were older, were more likely to be male, were more likely to have hypertension, and had higher HbA1c and hs-CRP.

\section{Cardiac Events During Follow-up}

The mean follow-up period was $2.8 \pm 1.4$ years (1246.4 personyears). The group without plaque did not develop any cardiac events during 675.1 person-years of follow-up. Among patients with subclinical atherosclerosis without significant stenosis $(\mathrm{n}=136)$ who were receiving medical treatment, 10 patients developed unstable angina pectoris and 1 patient developed AMI during 326.4 person-years of follow-up. Coronary angiograms were performed in these patients, and 9 patients underwent PCI according to angiographic findings.

Of the patients who had significant stenosis on cCTA, TMT was positive in 14 patients and negative in 70 . Patients who had positive TMT underwent conventional coronary angiography (CCA) and PCI or coronary artery bypass grafting (mean follow-up duration was $2.8 \pm 1.3$ years, or 39.2 person-years). Patients who had a negative TMT received medical treatment. Twenty-seven of them developed unstable angina pectoris or AMI during 203.2 person-years of follow-up. Coronary angiography was performed in those patients, and 21 patients were treated with PCI (Figure 1). On Kaplan-Meier curves for car- 


\begin{tabular}{|c|c|c|c|c|}
\hline \multirow{2}{*}{ Variable } & \multicolumn{2}{|c|}{ Unadjusted model } & \multicolumn{2}{|c|}{ After adjustment } \\
\hline & OR $(95 \% \mathrm{Cl})$ & P-value & OR $(95 \% \mathrm{Cl})$ & P-value \\
\hline \multicolumn{5}{|c|}{ Subclinical atherosclerosis } \\
\hline $\mathrm{hs}-\mathrm{CRP} \geq 2.0 \mathrm{mg} / \mathrm{L}$ & $6.458(3.662-11.386)$ & $<0.001$ & $6.743(3.437-13.228)$ & $<0.001$ \\
\hline Age $>60$ years & $1.631(1.113-2.391)$ & 0.012 & $2.384(1.315-4.322)$ & 0.004 \\
\hline Hypertension & $1.536(1.039-2.271)$ & 0.031 & $1.868(1.044-3.344)$ & 0.035 \\
\hline Male & $1.779(1.158-2.732)$ & 0.008 & $1.678(0.946-2.976)$ & 0.077 \\
\hline Current smoking & $2.438(1.500-3.961)$ & $<0.001$ & $1.759(0.885-3.497)$ & 0.107 \\
\hline High FRS group & $2.494(1.536-4.049)$ & $<0.001$ & $1.330(0.575-3.080)$ & 0.505 \\
\hline $\mathrm{HbA}_{1 \mathrm{c}} \geq 7.0 \%$ & $1.237(0.785-1.950)$ & 0.359 & $1.189(0.647-2.185)$ & 0.577 \\
\hline \multicolumn{5}{|c|}{ Significant coronary stenosis } \\
\hline $\mathrm{hs}-\mathrm{CRP} \geq 2.0 \mathrm{mg} / \mathrm{L}$ & $3.732(2.226-6.256)$ & $<0.001$ & $4.670(2.406-9.065)$ & $<0.001$ \\
\hline Hypertension & $1.872(1.157-3.030)$ & 0.010 & $2.517(1.255-5.048)$ & 0.009 \\
\hline Age $>60$ years & $1.654(1.026-2.668)$ & 0.038 & $2.495(1.194-5.214)$ & 0.015 \\
\hline Male & $2.024(1.092-3.745)$ & 0.023 & $1.939(0.912-4.122)$ & 0.085 \\
\hline $\mathrm{HbA}_{1 \mathrm{c}} \geq 7.0 \%$ & $0.994(0.557-1.773)$ & 0.984 & $1.423(0.668-3.032)$ & 0.361 \\
\hline Current smoking & $1.788(1.038-3.079)$ & 0.034 & $1.391(0.639-3.027)$ & 0.406 \\
\hline High FRS & 2.365 (1.394-4.013) & 0.001 & $1.080(0.411-2.840)$ & 0.876 \\
\hline \multicolumn{5}{|l|}{ Clinical events } \\
\hline Hypertension & $2.580(1.436-4.638)$ & 0.001 & $2.758(1.258-6.049)$ & 0.011 \\
\hline $\mathrm{hs}-\mathrm{CRP} \geq 2.0 \mathrm{mg} / \mathrm{L}$ & $1.464(0.756-2.835)$ & 0.256 & $2.158(1.194-4.684)$ & 0.042 \\
\hline Current smoking & $2.884(1.558-5.337)$ & $<0.001$ & $1.847(0.784-4.349)$ & 0.160 \\
\hline Age $>60$ years & $1.452(0.813-2.596)$ & 0.206 & $1.607(0.697-3.707)$ & 0.266 \\
\hline $\mathrm{HbA}_{1 \mathrm{c}} \geq 7.0 \%$ & $0.705(0.364-1.363)$ & 0.297 & $1.487(0.650-3.401)$ & 0.347 \\
\hline Male & $1.834(0.865-3.891)$ & 0.109 & $1.475(0.609-3.576)$ & 0.389 \\
\hline High FRS & $2.321(1.242-4.337)$ & 0.007 & $1.177(0.391-3.549)$ & 0.772 \\
\hline
\end{tabular}

$\mathrm{Cl}$, confidence interval; FRS, Framingham risk score; OR, odds ratio. Other abbreviations as in Table 1.

diac event-free survival rate, the highest event rate was seen in the subclinical atherosclerosis with significant stenosis group ( $\log$ rank $\mathrm{P}<0.001$; Figure 2). The incidence of cardiac death in the present study was $0 \%$ over 3 years of follow-up. The incidence of the primary endpoint increased as the total coronary artery calcium score and FRS increased (Figure 3).

\section{Predictors of MDCT Findings and Cardiac Events}

Multivariate logistic regression analysis was performed to identify the predictors of MDCT findings and cardiac events during follow-up. hs-CRP $\geq 2.0 \mathrm{mg} / \mathrm{L}$, age $>60$ years, and hypertension were independent predictors of subclinical atherosclerosis and significant coronary stenosis on MDCT. Also, hs-CRP $\geq 2.0 \mathrm{mg} / \mathrm{L}$ and hypertension were independent predictors of cardiac events after adjustment for the other variables (Table 3).

\section{Discussion}

In the current study, we investigated the usefulness of cardiac MDCT and TMT for identifying subclinical coronary atherosclerosis in asymptomatic patients with type 2 diabetes. The major finding was that this new strategy using cardiac MDCT and TMT can be useful for screening high-risk patients and minimizing mortality from cardiac death with appropriate early treatment before symptom manifestation.

The cardiovascular prognosis is worse in diabetic than in non-diabetic patients with cardiovascular disease, so much so that diabetes has been labeled a "coronary heart disease equivalent". ${ }^{12}$ Not only is CAD more extensive in patients with diabetes but it also has an atypical presentation, with myocardial ischemia manifesting without pain or any symptoms in a significant proportion of cases. Therefore, screening diabetic patients for heart disease and silent ischemia is critical for improving survival.

Although CCA is the gold standard test for diagnosing CAD, it is not suitable as a screening test in asymptomatic subjects because of its invasiveness and associated complications. Therefore, non-invasive screening methods are currently recommended for diabetic patients with a significant coronary risk factor profile. ${ }^{13}$ Cardiac MDCT is almost as good as invasive CCA in terms of detecting coronary artery stenosis and it has been used as a non-invasive screening method in the early detection of heart disease. ${ }^{14}$ MDCT does have several disadvantages, however, such as a tendency to overestimate the degree of stenosis compared with that estimated on $\mathrm{CCA} ;{ }^{15}$ high cost; and high radiation dose. Taking into account clinical considerations and the results of initial clinical trials, MDCT may be of particular utility in situations that require $\mathrm{CAD}$ to be reliably ruled out even though the pre-test likelihood for disease is not high. ${ }^{7}$ The role of MDCT for routine medical examination in asymptomatic diabetic patients has not been established. ${ }^{16}$

MDCT has limitations for identifying high-risk patients and for preventing adverse cardiac events when anatomical evaluation is used alone. Therefore, we added TMT as a functional evaluation. TMT is recommended in the current guideline, ${ }^{4}$ but some studies have reported that screening methods using TMT as the first-line method fail to detect some cases of asymptomatic CAD. ${ }^{5,6}$ Therefore, we suggested a new guideline for the management of asymptomatic diabetic patients in which the non-invasive, cost-effective, and widely available TMT is added for further management of diabetic patients with significant 
stenosis detected on MDCT. The result is worthy of close attention. The all-cause mortality rate was $0 \%$ in the present subjects during 3 years of follow-up. Also, the 84 patients who had significant stenosis of the coronary artery underwent TMT, which produced only 14 positive results. These 14 patients were direct candidates for CCA or PCI. During followup, among the 70 patients with negative TMT who received medical treatment, 27 patients had cardiac events, although only 11 cardiac events were detected during the same period in the group with insignificant stenosis. These results support the idea that both anatomical and functional assessment should be used in the evaluation of CAD. When we consider both anatomical and functional MDCT and TMT, effective screening for detection of subclinical atherosclerosis and reduction of adverse cardiac events can be achieved.

Some aspects of the methodology and results compared with previous studies should be considered. First, we included relatively high-risk patients who had concomitant cardiovascular disease risk factors such as hypertension, dyslipidemia, smoking, old age, and diabetes. A total of 323 subjects (72.5\%) belonged to the intermediate or high FRS group. One study suggested that once subclinical atherosclerosis has developed, it becomes the primary determinant of the risk of clinical CAD. ${ }^{17}$ Therefore, established CAD risk factors in these patients are important causative factors for development of atherosclerosis in diabetic subjects. Second, we found subclinical atherosclerosis in almost half of the cases. Significant stenosis of the coronary artery was found in $18.9 \%$ of cases, which reflects the importance of identification of unknown CAD in halting the progression of CAD. This finding agrees with those of previous studies using 64-slice MDCT. ${ }^{18,19}$ Using MDCT, Iwasaki et al identified CAD in $33.3 \%$ of asymptomatic diabetic patients, but that study lacked follow-up and functional evaluation of stenotic lesions. ${ }^{18}$

Third, the incidence of cardiac events increased as coronary artery calcium score (CACS) increased. CACS has been reported to predict all-cause mortality and cardiovascular events. ${ }^{20,21}$ The present study showed that $29.8 \%$ of total cardiac events occurred in the group with CACS 101-300, and $48.3 \%$ occurred in the group with CACS $>300$ during 3 years of follow-up. Viewed the other way, CACS has high negative predictive value. Some prospective studies showed that no events developed in subjects with CACS 0 during 2-3 years of follow-up. ${ }^{20,22}$ In the present study, also, there were no cardiac events in patients without subclinical atherosclerosis and CACS 0 during 3 years of follow-up. Therefore, we reaffirm the high sensitivity and low false-negative rate of MDCT, showing that MDCT is a useful test for excluding patients without CAD. And, given that many diabetic patients have chronic kidney disease, CACS has an advantage over CTA as a screening tool, because contrast agents are not required. ${ }^{23}$

Fourth, we also found that cardiac events increased according to FRS. Among patients in the low-risk FRS group, 3.3\% experienced cardiac events, compared with $12.9 \%$ in the intermediate-risk group and $19.8 \%$ in the high-risk group. Moreover, there was a linear correlation with CACS and FRS. Thus, it is reasonable to select patients by this traditional risk assessment method according to CACS. The present study showed that in high-risk patients with unknown CAD, as in the case of the present diabetic patients, MDCT often shows severe coronary atherosclerosis.

In the present study, high hs-CRP $\geq 2.0 \mathrm{mg} / \mathrm{dl}$ was an independent predictor of subclinical atherosclerosis, significant stenosis, and cardiac events. A previous study showed that hs-CRP elevation is associated with cardiovascular events in the healthy general population. ${ }^{24} \mathrm{We}$ also found that hs-CRP was higher in the group with subclinical atherosclerosis than in the group without subclinical atherosclerosis, and in the group with significant stenosis than in the group with insignificant stenosis.

Another biomarker, $\mathrm{HbA}_{1 \mathrm{c}}$, has also been associated with clinical cardiovascular disease in both diabetic and non-diabetic subjects. ${ }^{25}$ In the present study, HbA1c was higher in the group with significant stenosis than in the group with insignificant stenosis, but it was not an independent predictor of subclinical atherosclerosis or significant stenosis on multivariate logistic regression analysis.

The present study had some limitations. First, the study patients were all self-referred, which is a limitation because of the patients' awareness of their condition. Second, we did not evaluate clinical or CT parameters in subjects who did not have diabetes. In other words, the present study lacked a control group. Third, we did not consider the cost-effectiveness of the strategy compared with other tests. Fourth, we did not evaluate changes in fasting glucose and $\mathrm{HbA}_{1 \mathrm{c}}$ level in each group at follow-up. Finally, we did not evaluate the long-term adverse effects of radiation exposure from cardiac MDCT.

\section{Conclusions}

This new strategy to detect subclinical atherosclerosis according to anatomical evaluation with cardiac MDCT and functional evaluation with TMT may be a useful method for minimizing the mortality rate from cardiovascular disease in asymptomatic diabetic patients.

\section{Acknowledgments}

This study was supported by a grant of the National Research Foundation of Korea Grant funded by the Korean Government (MEST), Republic of Korea (2010-0020261), and the Korean Health Technology R\&D Project, Ministry of Health \& Welfare, Republic of Korea (A120247). None of the authors have or perceived a conflict of interest.

\section{References}

1. Wong ND, Nelson JC, Granston T, Bertoni AG, Blumenthal RS, Carr $\mathrm{JJ}$, et al. Metabolic syndrome, diabetes, and incidence and progression of coronary calcium: The multiethnic study of atherosclerosis study. JACC Cardiovasc Imaging 2012; 5: 358-366.

2. Zbinden R, Wenger U, Meier P, Gloekler S, Windecker S, Meier B, et al. Silent ischemia normalized for coronary collateral function in patients with and without diabetes mellitus. Int J Cardiol 2011; 147: 319-321.

3. Rajagopalan N, Miller TD, Hodge DO, Frye RL, Gibbons RJ. Identifying high-risk asymptomatic diabetic patients who are candidates for screening stress single-photon emission computed tomography imaging. J Am Coll Cardiol 2005; 45: 43-49.

4. American Diabetes Association. Consensus development conference on the diagnosis of coronary heart disease in people with diabetes. Diabetes Care 1998; 21: 1551-1559.

5. Scognamiglio R, Negut C, Ramondo A, Tiengo A, Avogaro A. Detection of coronary artery disease in asymptomatic patients with type 2 diabetes mellitus. J Am Coll Cardiol 2006; 47: 65-71.

6. Kawasaki-Ogita Y, Hamamoto Y, Honjo S, Ikeda H, Wada Y, Nomura $\mathrm{K}$, et al. The limited usefulness of the treadmill test or a risk-guided approach in screening for asymptomatic coronary heart disease in Japanese patients with type 2 diabetes. Intern Med 2012; 51: 3337 3342 .

7. Schroeder S, Achenbach S, Bengel F, Burgstahler C, Cademartiri F, de Feyter P, et al. Working Group Nuclear Cardiology and Cardiac CT; European Society of Cardiology; European Council of Nuclear Cardiology. Cardiac computed tomography: Indications, applications, limitations, and training requirements: Report of a Writing Group deployed by the Working Group Nuclear Cardiology and Cardiac CT of the European Society of Cardiology and the European Council of Nuclear Cardiology. Eur Heart J 2008; 29: 531-556.

8. Agatston AS, Janowitz WR, Hildner FJ, Zusmer NR, Viamonte M 
Jr, Detrano R. Quantification of coronary artery calcium using ultrafast computed tomography. J Am Coll Cardiol 1990; 15: 827-832.

9. Weigold WG, Abbara S, Achenbach S, Arbab-Zadeh A, Berman D, Carr JJ, et al. Standardized medical terminology for cardiac computed tomography: A report of the Society of Cardiovascular Computed Tomography. J Cardiovasc Comput Tomogr 2011; 5: 136-144.

10. Raff GL, Abidov A, Achenbach S, Berman DS, Boxt LM, Budoff MJ, et al. Society of Cardiovascular Computed Tomography: SCCT guidelines for the interpretation and reporting of coronary computed tomographic angiography. J Cardiovasc Comput Tomogr 2009; 3: 122 136.

11. Hausleiter J, Meyer T, Hadamitzky M, Kastrati A, Martinoff S, Schömig A. Prevalence of noncalcified coronary plaques by 64-slice computed tomography in patients with an intermediate risk for significant coronary artery disease. J Am Coll Cardiol 2006; 48: 312 318.

12. Valensi P. Silent myocardial ischaemia. To screen or not to screen? That is... Arch Cardiovasc Dis 2008; 101: 509-511.

13. Falcone C, Nespoli L, Geroldi D, Gazzaruso C, Buzzi MP, Auguadro $\mathrm{C}$, et al. Silent myocardial ischaemia in diabetic and nondiabetic patients with coronary artery disease. Int J Cardiol 2003; 90: 219-227.

14. Motoyama S, Sarai M, Inoue K, Kawai H, Ito H, Harigaya $\mathrm{H}$, et al. Morphologic and functional assessment of coronary artery disease: Potential application of computed tomography angiography and myocardial perfusion imaging. Circ J 2013; 77: 411-417.

15. Voros S, Rinehart S, Qian Z, Joshi P, Vazquez G, Fischer C, et al. Coronary atherosclerosis imaging by coronary $\mathrm{CT}$ angiography: Current status, correlation with intravascular interrogation and metaanalysis. JACC Cardiovasc Imaging 2011; 4: 537-548.

16. Fujimoto S, Kondo T, Kodama T, Orihara T, Sugiyama J, Kondo M, et al. Coronary computed tomography angiography-based coronary risk stratification in subjects presenting with no or atypical symptoms. Circ J 2012; 76: 2419-2425.

17. Kuller LH, Velentgas P, Barzilay J, Beauchamp NJ, O'Leary DH, Savage PJ. Diabetes mellitus: Subclinical cardiovascular disease and risk of incident cardiovascular disease and all-cause mortality. Arterioscler Thromb Vasc Biol 2000; 20: 823-829.

18. Iwasaki K, Matsumoto T, Aono H, Furukawa H, Samukawa M. Prevalence of subclinical atherosclerosis in asymptomatic diabetic patients by 64-slice computed tomography. Coron Artery Dis 2008; 19: $195-201$.

19. Kaiser C, Bremerich J, Haller S, Brunner-La Rocca HP, Bongartz G, Pfisterer M, et al. Limited diagnostic yield of non-invasive coronary angiography by 16 -slice multi-detector spiral computed tomography in routine patients referred for evaluation of coronary artery disease. Eur Heart J 2005; 26: 1987-1992.

20. Hou ZH, Lu B, Gao Y, Jiang SL, Wang Y, Li W, et al. Prognostic value of coronary $\mathrm{CT}$ angiography and calcium score for major adverse cardiac events in outpatients. JACC Cardiovasc Imaging 2012; 5: $990-999$.

21. Li J, Flammer AJ, Nelson RE, Gulati R, Friedman PA, Thomas RJ, et al. Normal vascular function as a prerequisite for the absence of coronary calcification in patients free of cardiovascular disease and diabetes. Circ J 2012; 76: 2705-2710.

22. Arad Y, Spadaro L, Goodman K, Newstein D, Guerci AD. Prediction of coronary events with electron beam computed tomography. J Am Coll Cardiol 2000; 36: 1253-1260.

23. Nakajima K, Matsuo S, Okuyama C, Hatta T, Tsukamoto K, Nishimura $\mathrm{S}$, et al. Cardiac event risk in Japanese subjects estimated using gated myocardial perfusion imaging, in conjunction with diabetes mellitus and chronic kidney disease. Circ J 2012; 76: 168-175.

24. Ridker PM, Hennekens CH, Buring JE, Rifai N. C-reactive protein and other markers of inflammation in the prediction of cardiovascular disease in women. $N$ Engl J Med 2000; 342: 836-843.

25. Tandjung K, van Houwelingen KG, Jansen H, Basalus MW, Sen H, Löwik MM, et al. Comparison of frequency of periprocedural myocardial infarction in patients with and without diabetes mellitus to those with previously unknown but elevated glycated hemoglobin levels (from the TWENTE Trial). Am J Cardiol 2012; 110: 1561-1567. 